\title{
A Study and Design of a Rat-Race Coupler Based Microwave Mixer
}

\author{
Asif Ahmed \\ Department of EEE, American International University Bangladesh, Dhaka, Bangladesh \\ Email address: \\ aahmed@aiub.edu \\ To cite this article: \\ Asif Ahmed. A Study and Design of a Rat-Race Coupler Based Microwave Mixer. Journal of Electrical and Electronic Engineering. \\ Vol. 3, No. 5, 2015, pp. 121-126. doi: 10.11648/j.jeee.20150305.14
}

\begin{abstract}
A microwave mixer circuits has been designed and fabricated, having frequency range of $2 \mathrm{GHz}-4 \mathrm{GHz}$, with optimum operating point at $3 \mathrm{GHz}$. The underlying theory of rat-race coupler was used to achieve frequency up-conversion and down-conversion process with the help of a nonlinear element - Schottky diode. The simulation of both the rat-race coupler and the mixer circuit has been done with Agilent Advanced Design System (ADS) software, and the fabricated mixer circuit has been tested using Vector Spectrum Analyzer (VSA). The measured results of the mixer has shown perfect consistency with the simulated outputs.
\end{abstract}

Keywords: Rat-Race Coupler, Microwave Mixer, Non-Linear Mixing

\section{Introduction}

A microwave mixer [1-8] is a device that up-concerts low frequency signal to microwave signal, and down-converts microwave signal to a corresponding base-band signal. The most usual application of mixers is in transceiver where two frequency beat together to generate two different frequencies, i.e., sum and difference of the two frequencies.

The history of mixers dates back to 1900 s when it was used for radio reception circuits. During the time of Second World War microwave mixers were very commonly used for military radar. Now-a-days, microwave mixers gained its interest for both civil and military purposes, for example, radars, transceivers for WLAN, satellite, guided weapon and radio astronomy, transportation, etc. The application of the microwave mixers ranges from one to several hundred gigahertz. Research and Development (R\&D) labs has been improvised microwave mixers by shifting from simple lumped components deployment to incorporation of nonlinear components, e.g., diodes. The continuous endeavor has been progressed through the traditional lumped component approach to the hybrid microwave integrated circuits (MIC) and the monolithic microwave integrated circuit (MMIC). The challenges of suppressing intermodulation products (IP) has been an important characteristic attributes of more complex mixer circuits.

This paper will show a design and fabrication of a mixer with Schottky diode as nonlinear component, and the corresponding measurement results to compare with simulated expectations.

\section{Theory Behind Frequency Mixers}

The effort of development of mixers has been given us many variations [9] in the kind of mixers circuits.

Single-Ended Mixer (SEM): is the most preliminary form of mixer that has only one port to mix where the signal are combined externally to feed to it.

Single-Balanced Mixer (SBM): combines two single ended mixers, and local oscillator (LO) noise side-band is balanced out by via a 4-port $3 \mathrm{~dB}$ coupler.

Double-Balanced Mixer (DBM): incorporates two baluns and mixing components as many as four in quantity in the form of a ring, bridge, star or quad. Inherently, it cancels LO amplitude modulated (AM) noise, and has high bandwidth.

Double-Double Balanced Mixer (DDBM): has eight mixing components and it separates RF, LO and intermediate frequency (IF) rendering improved (higher) dynamic range.

Image Rejection Mixer (IRM): employs two identical mixers either SEM, SBM or DBM in a phase alignment so as to reject the images.

Image Recovery (Enhancement) Mixer: is a kind of IRM where image power from one mixer is converted to IF in other mixer, and vice versa.

Researches have been conducted to improve linearity [10] 
of a mixer. By the term linearity, it means that the input and output changes should have same value. The linearity is also an important attribute of mixers as without linearity there would be unwanted AM noise at the output.

Another common attribute of a mixer is its conversion efficiency, and many efforts have been expended in making the conversion efficiency (CE) [11] of a mixer optimum. The conversion efficiency is the difference between the RF input power and IF output power.

$$
C E=R F \text { Power }(d B m)-I F \text { Power }(d B m) .
$$

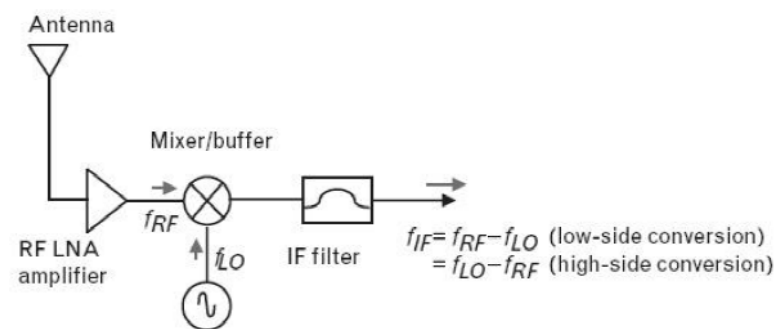

Figure 1. Basic function of a mixer.

In general, it can be described briefly about mixer that it is a non-linear transducer having 3-ports, namely, RF (Radio Frequency) port, LO (Local Oscillator) port and IF (Intermediate Frequency) port. The RF is the transmission frequency that is converted to IF for the purpose of improved selectivity. Figure 1 shows a basic functional mixer employed in a receiver.

Another attribute of a frequency mixer is its image frequency (IM), and in [12], it shown how to characterize a mixer by 3-port conversion matrix for RF, IF and IM signals.

At the IF port of mixer all frequencies: $\omega= \pm\left(N . \omega_{L O} \pm\right.$ $\left.M . \omega_{R F}\right)$ can exist. The general expression of IF for down conversion is following:

$$
\omega_{I F}= \pm\left(\omega_{L O}-\omega_{R F}\right)
$$

Conversely, the expression of up-conversion is

$$
\omega_{I F}= \pm\left(\omega_{L O}+\omega_{R F}\right)
$$

The IF and IM signals at the IF port for the downconversion are defined as follows:

$$
\begin{aligned}
& \omega_{I F}=\omega_{R F}-\omega_{L o}, \\
& \omega_{I M}=\omega_{R F}+\omega_{L o} .
\end{aligned}
$$

Recent literature [13-15] shows other attributes of mixers with different working principles.
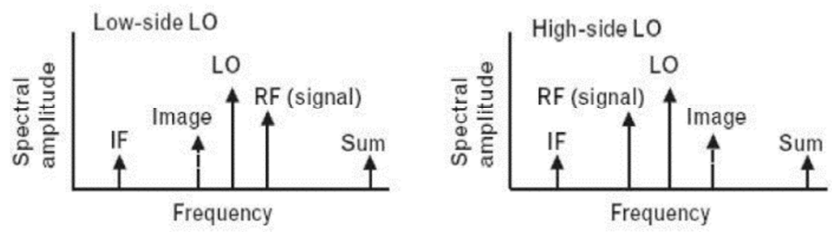

Figure 2. Converted frequency spectrum.
Figure 2 shows sketches of converted frequencies in frequency domain.

Figure 3 illustrates the block diagram of a harmonic mixer. In this paper a rat-race coupler has been used for adding and subtracting the signals and diode for implementing the nonlinear block.

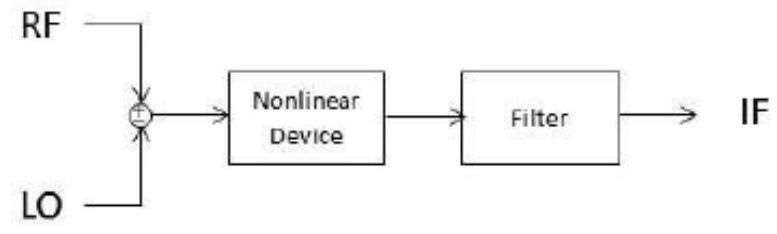

Figure 3. Harmonic Mixer.

Harmonic mixers produce more than just the sum and difference frequencies. The intermodulation products are given by $=N \times R F \pm M \times L O$, and their levels, relative to the desired output of $R F \pm L O$, for a common mixer.

\subsection{Rat-Race Coupler}

Rat-race coupler is a $3 \mathrm{~dB}$ coupler consists of four transmission lines with impedance of $\sqrt{2} Z_{0}$, three with length of $\lambda / 4$ and one with length of $3 \lambda / 4$. There are four terminals with impedance of $Z_{0}$.

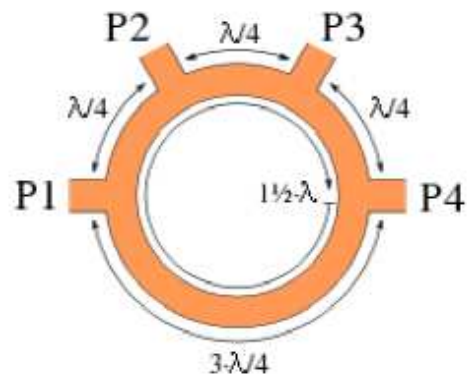

Figure 4. Rat-race coupler.

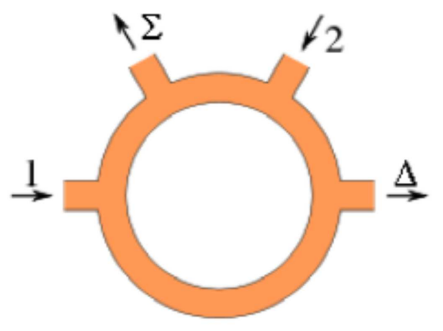

Figure 5. Function of the rat-race ports.

Figure 4 depicts a basic form of a rat-race coupler. The Sparameter matrix of the coupler is following:

$$
S=-\frac{j}{\sqrt{2}}\left[\begin{array}{cccc}
0 & 1 & 0 & -1 \\
1 & 0 & 1 & 0 \\
0 & 1 & 0 & 1 \\
-1 & 0 & 1 & 0
\end{array}\right]
$$

This coupler can divide the input power in halves but the output waves are in 0 or 180 degree phase difference. If we 
apply two inputs in the first and third ports we would have the sum of the waves from the second, and differences in the fourth port as shown in figure 5 .

\subsection{Nonlinear Elements}

If we apply the sum and differences of the rat-race coupler to a nonlinear device, we would have different harmonics of their multiplications which is the base of the operation of harmonic mixers. By filtering out the undesired harmonics, we can have the demodulated signal from the carrier.

The diode used for mixing can be modeled at the RF frequency as a resistor and capacitor in parallel. The resistor is usually in a range of 50 to $150 \Omega$, and the capacitor between 1 and 1.5 times the junction capacitance. The IF output impedance is usually between $75 \mathrm{ohms}$ and $150 \mathrm{ohms}$. At low IF frequencies the output impedance is almost purely resistive. Therefore, a matching network between coupler and the diode is needed for the particular frequency in order to deliver the maximum power in that frequency. Well-matched diodes can reduce the overall noise figure (NF) too.

For selecting the mixing diode, one has to look for the cutoff frequency of the diode, for series resistance $R_{s}$ and junction capacitance $C_{j}$.

In order to ground the RF and LO signal in the output of nonlinear device these frequencies should be grounded by a small capacitor, and also it can damp the IF noise at the output.

\section{Design and Fabrication with ADS}

Schematics of Rat-race Coupler

The schematic of the rat-race coupler has been drawn in Agilent's ADS software. As we already know it includes four pieces of $\sqrt{2} Z_{0}$ transmission lines (three with $\lambda / 4$ and one with $3 \lambda / 4$ ) for the circle, 4 T-junctions and 4 pieces of $Z_{0}$ transmission line for the terminals. Multiple $\lambda / 4$ has been used for the case of $3 \lambda / 4$ and some of them are curved in order to have a shape close to the circle.

Figure 6 schematic part of the coupler part of the mixer. It includes the 4 ports and transmission lines that produce the coupler. Substrate parameters for micro-strip are also shown. The width and length of the transmission line has been calculated with LineCalc tool included in the ADS software.

Using S-parameter simulation, the plot of $S_{11}, S_{12}, S_{21}$ and $S_{22}$ has been obtained between the frequency $1 \mathrm{GHz}$ and $6 \mathrm{GHz}$ as shown in figure 7. As depicts in the figure 7, for $S_{11}$ and $S_{12}$ there is more than $40 \mathrm{~dB}$ attenuation, and for the other ports the attenuation is about $4 \mathrm{~dB}$. Comparing the $\mathrm{S}$ parameter matrix for rat-race, $-40 \mathrm{~dB}$ represents the zero and $-4 \mathrm{~dB}$ represents 1 . The layout of the rat-race is shown in figure 8 .

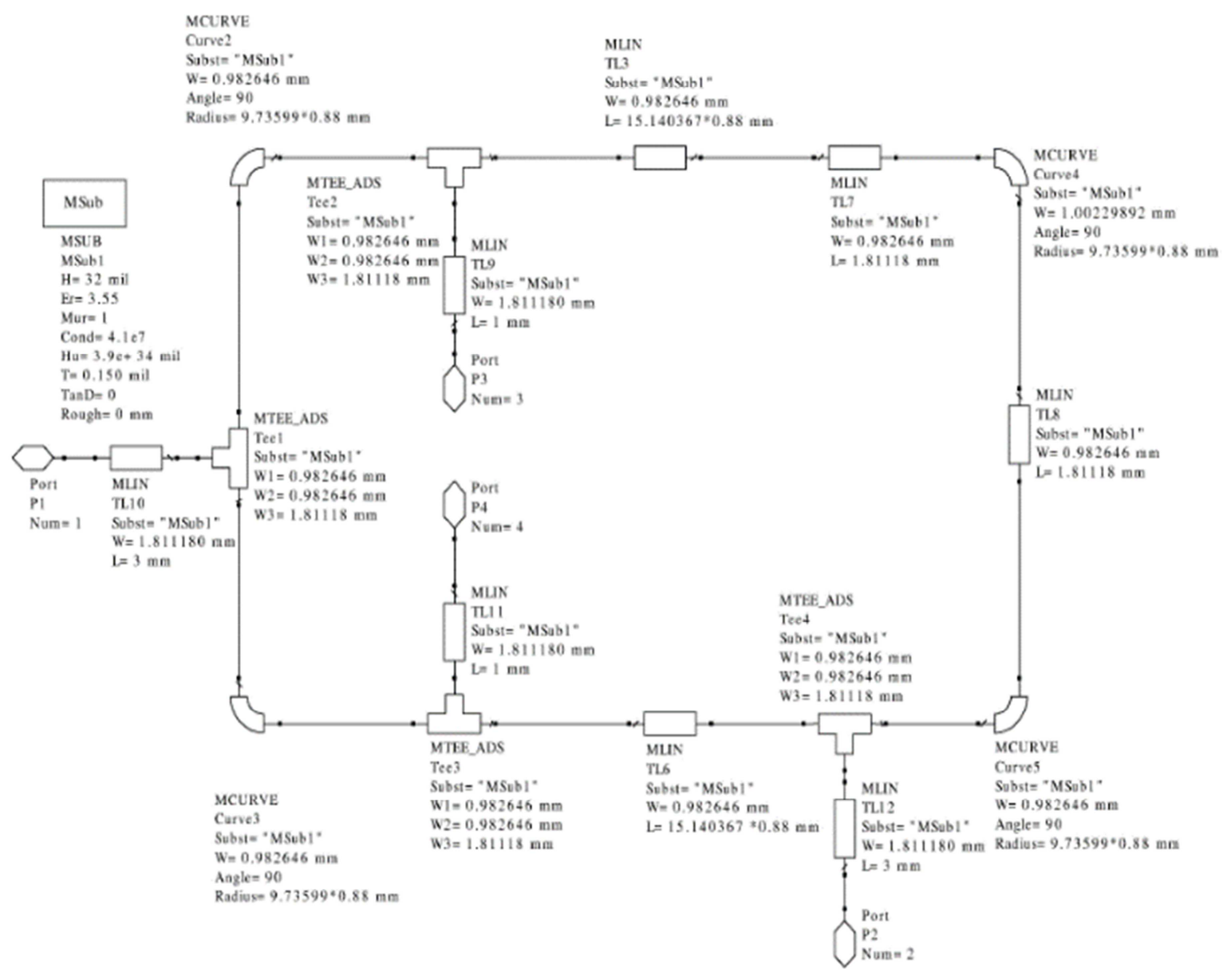

Figure 6. Rat-race Schematic. 


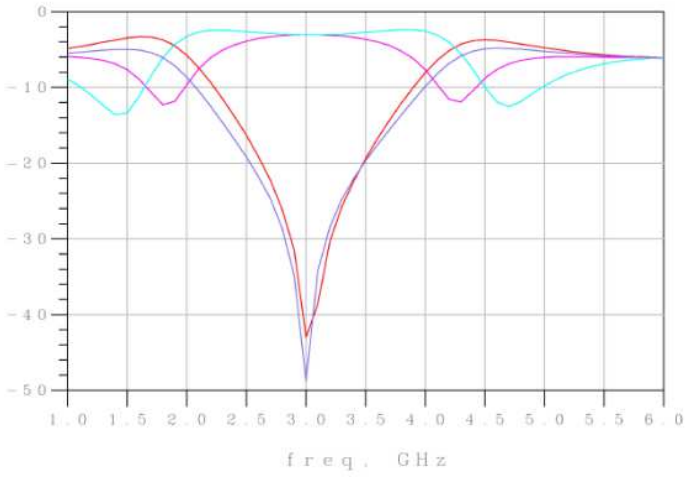

Figure 7. Rat-race S-parameter plot.

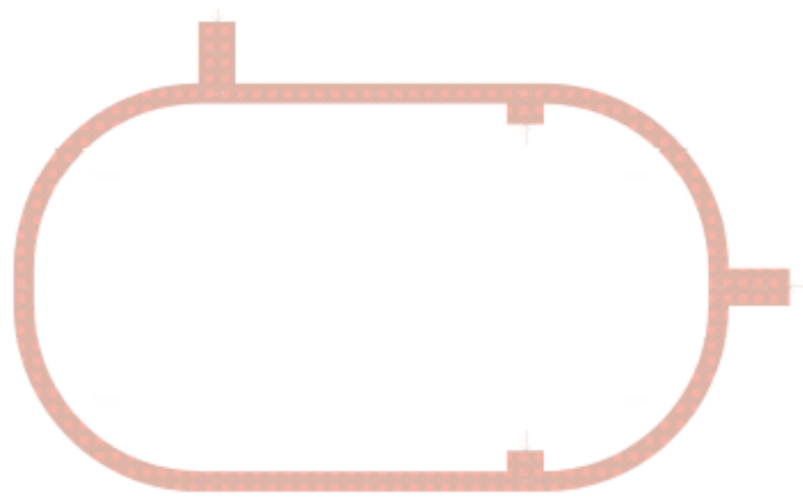

Figure 8. Rat-race layout.

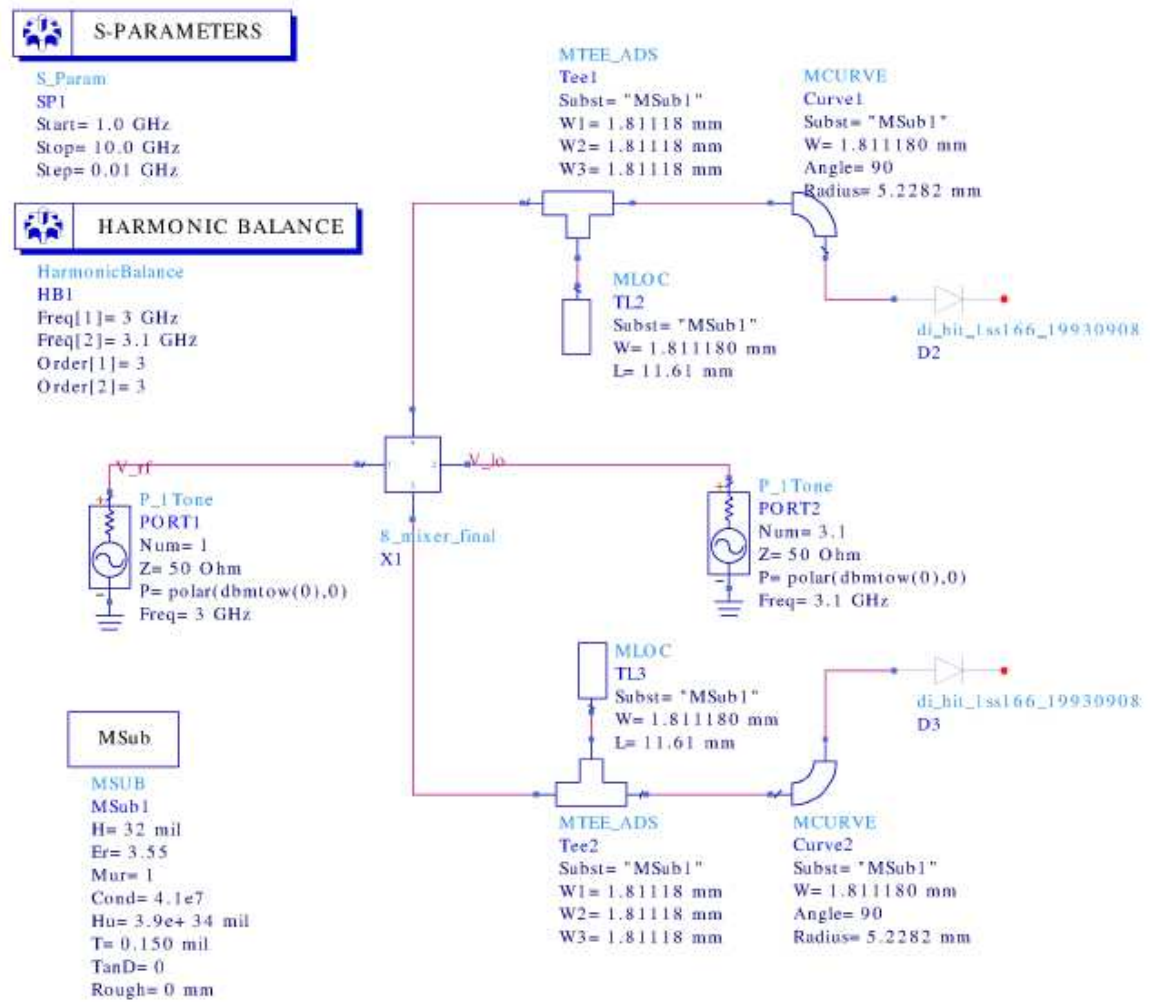

Figure 9. Complete Mixer Schematic.

After this the nonlinear components, i.e., diodes were added for achieving the frequency mixing, but since the input impedance of diode is not $50 \Omega$, so we need to design matching network. Complete schematic including the rat-race and the matching network along with the nonlinear devices is presented in Figure 9.

Figure 10 shows the final layout of the complete mixer which later were fabricated with chemical etching process, and were tested for conformity to the simulated output.

The simulation of the S-parameters has been set between 1 $\mathrm{GHz}$ to $10 \mathrm{GHz}$ with $0.01 \mathrm{GHz}$ steps.

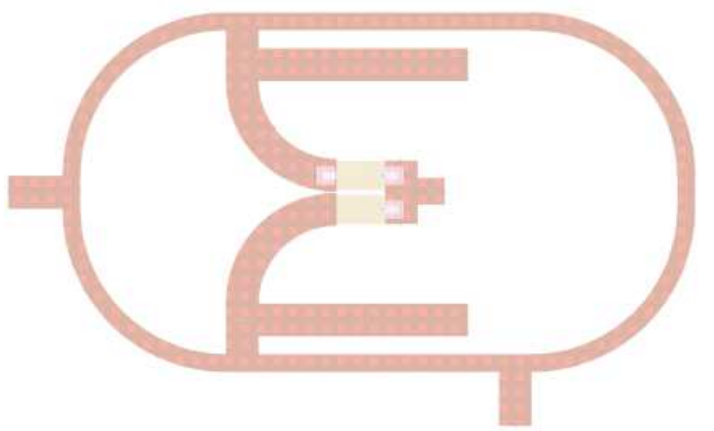

Figure 10. Complete Mixer layout. 


\section{Results: Fabrication and Measurement}

The layout has been obtained from the ADS, and was saved in GDSII file format for it to be opened in LayoutEditor software which gives us the actual size of the layout graphics by printing it in pdf file. Using the printed graphics of mixer layout, a mask was created to develop the circuit on the copper clad board which later on was etched to develop the complete mixer layout. Figure 11 shows the fabricated layout of the complete mixer.

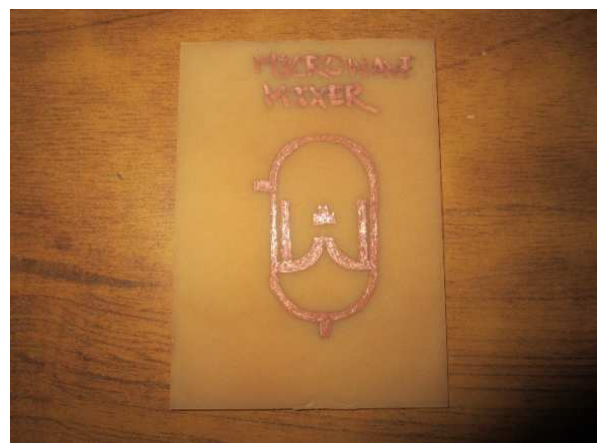

Figure 11. Fabricated Mixer layout on copper board.

The development of the complete circuit has been conducted by connecting and soldering the nonlinear components. Two SMD (Surface Mount Device) diodes were connected and soldered as shown in figure 12. Three PCB mount SMA-RF (jack) connector were connected to the RF, $\mathrm{LO}$ and IF ports.

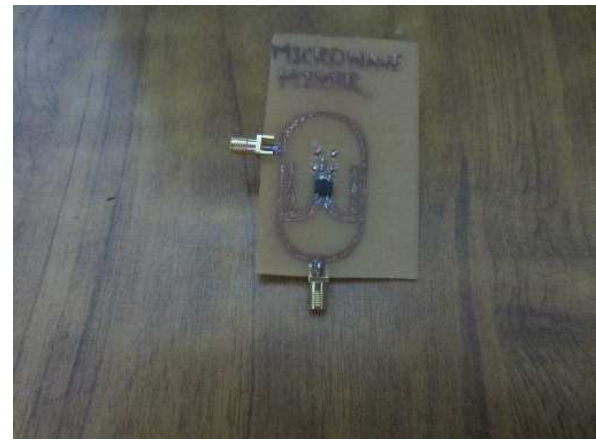

(a)

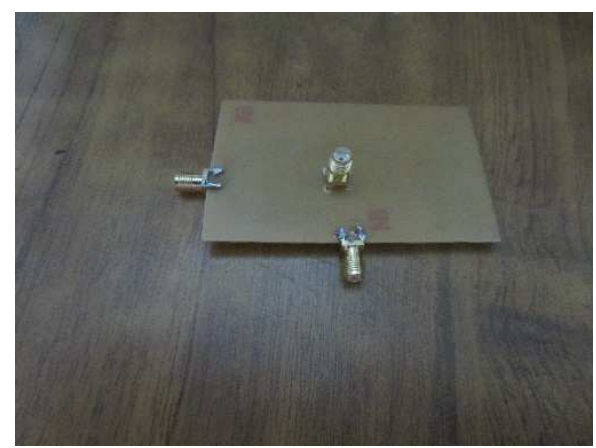

(b)

Figure 12. Completed Microwave Mixer.
For testing the developed mixer circuit, two signal has been applied in RF and LO port of frequency $3.1 \mathrm{GHz}$ and 3 $\mathrm{GHz}$ respectively. A $40 p F$ capacitor was used as LPF (Low Pass Filter) at the output. The output magnitude for both high and low frequency is presented in figure 13 and figure 14 respectively.

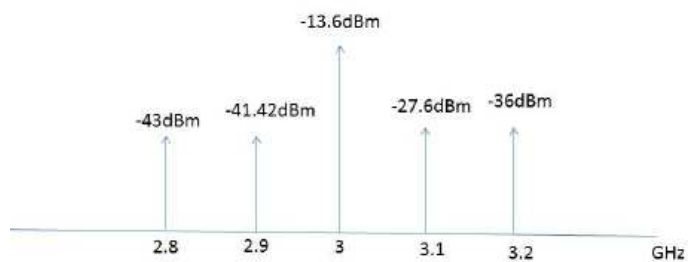

Figure 13. High Frequency output.

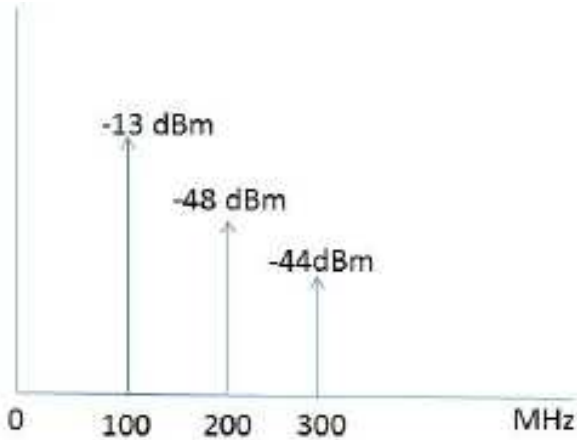

Figure 14. Low Frequency output.

The measured outputs from the spectrum analyzer is shown in the figure 15 and figure 16 for high and low frequency respectively.

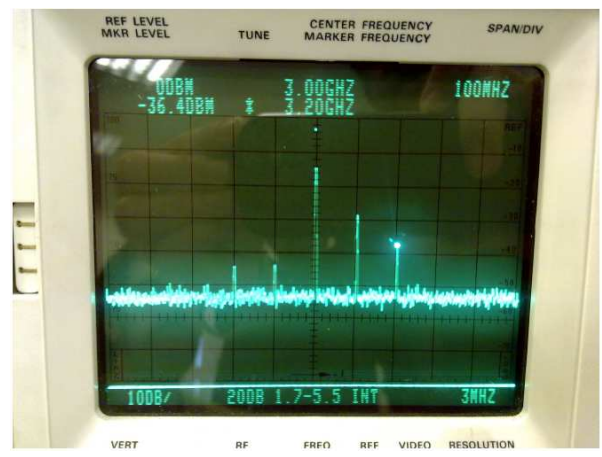

Figure 15. High Frequency Spectrum.

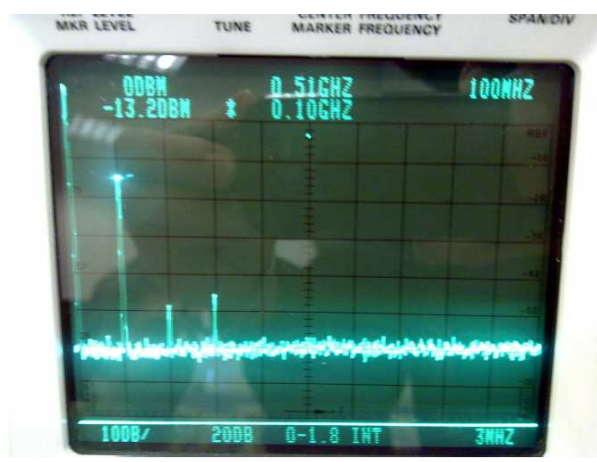

Figure 16. Low Frequency Spectrum. 
Furthermore, the RF port has been fed with an AM modulated signal with central frequency of $3.1 \mathrm{GHz}$ and modulating signal frequency of $200 \mathrm{kHz}$. The output was exactly as expected as shown in figure 17.

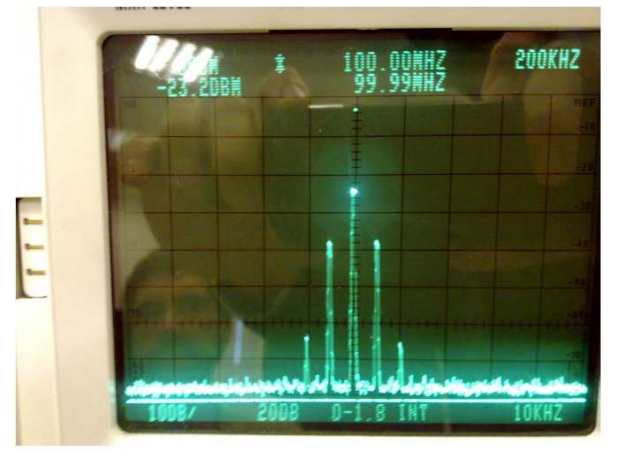

Figure 17. LF spectrum of down-converted modulated signal.

\section{Discussion and Conclusion}

The design of the microwave mixer involved designing and calculation of line length, width for the conformance to required line impedances, and LineCalc application integrated in ADS software was used carefully for that purpose.

The whole circuit needed to match to the nonlinear diodes that was applied at the end part of the design process. The output (IF) has been taken from the inside part of the coupler circle so as to make the whole device more compact and easy to handle.

For the design requirements T-connectors were used, and those were needed to be included carefully and calculatedly as they could have been changed the response of the coupler.

The resonant frequency of the whole circuit would change if the T-connectors, line width and lengths is changed or modified incorrectly.

\section{References}

[1] H. E. M. Barlow, K. V. G. Krishna, "A Hall-effect microwave mixer" in Proc. of IEE- Part B: Electronic and Communication Engineering, 1962, vol. 109, no. 44, pp. 131-136.

[2] H. J. O'Neill, "Image-frequency effects in a microwave crystal mixer," in Proc. of the Institution of Electrical Engineers, 1965, vol. 112, no. 11, pp. 2019-2024.

[3] T. Oxley, F. Hilsdent, "The performance of backward diodes as mixers and detectors at microwave frequencies" Radio and Electronic Engineer, 1966, vol. 31, no. 3, pp. 181-191.

[4] W. J. Moroney, Y. Anand, "Reliability of Microwave mixer diodes" in $10^{\text {th }}$ Annual Reliability Physics Symposium, 1972, pp. 57-63.

[5] O. S. A. Tang, C. S. Aitchison, "Practical performance of a microwave distributed MESFET mixer" Electronic Letters, 1985, vol. 21, no. 5, pp. 172-173.
[6] G. Tomassetti, "An Unsual microwave mixer" in $16^{\text {th }}$ European Microwave Conference, 1986, pp. 754-759.

[7] M. I. Sobhy, F. Bassirato, "Non-ideal modelling and design of microwave mixers" in IEEE MTT-S International Microwave Symposium Digest, 1988, pp. 1111-1114.

[8] H. Zaghloul, T. H. T. van Kalleveen, et. al., "A simple method for the evaluation of microwave mixer diodes" IEEE Transactions on Instrumentation and Measurement, 1990, vol. 39, no. 6, pp. 928-932.

[9] T. H. Oxley, "50 years development of the microwave mixer for heterodyne reception" IEEE Transactions on Microwave Theory and Techniques, 2002, vol. 50, no. 3, pp. 867-876.

[10] K. Holland, J. Howes, "Improvements to the microwave mixer and power sensor linearity measurement capability at the National Physical Laboratory" in IEE Proc.- Science, Measurement and Technology, 2002, vol. 149, no. 6, pp. 329-332.

[11] Gan Chee Tat, Lim Koon Tin, "A study and design of microwave mixer for Conversion Efficiency (CE) performance" in Asia-Pacific Conference on Applied Electromagnetics, 2007, pp. 1-5.

[12] Cao Haibo, R. Weber, "Three-port conversion scattering parameters characterization for microwave mixers" in $51^{\text {st }}$ Midwest Symposium on Circuits and Systems, 2008, pp. 414417.

[13] C. Bohemond, T. Rampone, A. Sharaiha, "Performance of a Photonic Microwave Mixer based on cross-gain modulation in a semiconductor optical amplifier" Journal of Lightwave Technology, 2011, vol. 29, no. 16, pp. 2402-2409.

[14] Yuding Wang, Lv Zhiqing, et. al., "Back door effects of High Power microwave on microwave mixer" in International Conference on Microwave and Millimeter Wave Technology (ICMMT), 2012, vol. 4, pp. 1-4.

[15] A. Kazemipour, M. Salhi, et. al., "Novel method to measure the conversion-losses (C.L.) of microwave and mm-wave mixers" in Asia-Pacific Microwave Conference Proc. (APMC), 2013, pp. 731-733.

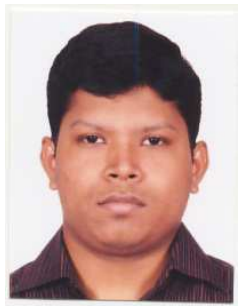

\section{Asif Ahmed}

Asif Ahmed received his B. S. degree in Electrical and Electronic Engineering from American International University Bangladesh in 2010, and M.S. degree in Wireless Communication from Jacobs University Bremen, Bremen, Germany, in 2012. He is currently working as a lecturer of Electrical and Communication Engineering in American International University Bangladesh. His research interests are wireless system development, RF device development and errorfree communication system. 